\title{
Equalização Cega via um Banco de Filtros de Erro de Predição Largamente Linear com Atraso de Equalização
}

\author{
Francisco J. A. de Aquino, Carlos A. F. da Rocha e Leonardo S. Resende
}

\begin{abstract}
Resumo - Neste artigo é apresentado um novo esquema de equalização robusta baseado em um banco de filtros de erro de predição largamente linear (FEPLL). As saídas destes FEPLLs são alinhadas para gerar uma estimativa atrasada mais confiável do símbolo transmitido. Esta estimativa é usada como seqüência de treinamento para um equalizador largamente linear. Como resultado, para uma relação sinal-ruído moderada ( $>15 \mathrm{~dB}$ ) é possível se obter uma taxa de erro de símbolo equivalente a que seria obtida por uma equalização de canal treinada. $O$ equalizador proposto é baseado no processamento largamente linear, aplicável quando o sinal transmitido é impróprio.
\end{abstract}

Palavras-Chave - Algoritmo LMS, filtragem adaptativa, processamento largamente linear, equalização cega.

Abstract - In this paper, we present a new robust blind equalizer scheme based on widely linear prediction error filter (WLPEF) bank. The WLPEF outputs are aligned to generate a more delayed reliable estimate of the transmitted symbol. This symbol estimate is used as training sequence for an WL equalizer. As result, for a moderated SNR ( $>15 \mathrm{~dB}$ ), we can obtain a symbol error rate equivalent that it would be obtained by a trained channel equalization. The proposed equalizer is based on the widely linear processing, applicable when the transmitted signal is improper.

Keywords - Adaptive filtering, LMS algorithm, widely linear processing, blind equalizer.

\section{INTRODUÇÃO}

Em geral, os canais digitais de comunicação são limitados em banda e estão sujeitos a ruídos e interferências. Estas limitações no canal de comunicação levam à interferência entre os símbolos (IES) na seqüência de dados captados no receptor. Para combater os efeitos desta distorção pode-se usar um equalizador [1].

As técnicas convencionais de equalização e recuperação de portadora usam seqüências de treinamento que são periodicamente retransmitidas. Como uma conseqüência imediata, ocorre uma redução na taxa de transmissão dos dados. Em algumas situações, entretanto, não é possível transmitir uma seqüência de treinamento para ajustar os parâmetros do equalizador.

Este trabalho foi parcialmente financiado pelo Centro Federal de Educação Tecnológica do Ceará (CEFET-Ce), Universidade Federal de Santa Catarina (UFSC) e Conselho Nacional de Desenvolvimento Científico e Tecnológico (CNPq) processo número 484391/2006-2.

F. J. A. de Aquino é professor no CEFET-Ce e aluno de doutorado na UFSC no Grupo de Pesquisa em Comunicação (GPqCom), e-mail: fcoalves_aq@cefet-ce.br. C. A. da Rocha e L. S. Resende são professores no Departamento de Engenharia Elétrica da UFSC, laboratório GPqCom. Emails: \{aurelio, leonardo\}@eel.ufsc.br.
Assim, os próprios dados transmitidos (desconhecidos) são usados para "treinar" o equalizador. Tem-se, neste caso, uma equalização cega ou autodidata do canal.

Vários pesquisadores, nos últimos anos, têm proposto o uso do processamento largamente linear como uma forma de melhorar o desempenho de equalizadores [2 - 5], quando o sinal recebido é impróprio [6]. Um exemplo de sinal impróprio ocorre quando um sinal real (sinal pertencente a uma constelação PAM, por exemplo) é transmitido por um canal complexo. Outros exemplos importantes são o caso de uma transmissão OQAM (offset quadrature amplitude modulation) e GSM (Global System for Mobile Communication), como mostrado em [3].

Recentemente, o uso de um filtro de erro de predição largamente linear (FEPLL) para a equalização cega de canais de comunicação com uma baixa complexidade computacional foi proposto pelos autores em [5]. Esta técnica, inerentemente, apresenta atraso zero, ou seja, o símbolo na saída do equalizador no instante discreto $k$ é uma estimativa do símbolo transmitido no instante $k$. Isto, entretanto, pode não ser a melhor solução, especialmente quando o canal considerado é de fase não mínima. A seleção de um atraso mais apropriado, que reflita a distribuição de energia do canal, pode resultar em um melhor desempenho do equalizador. Uma solução encontrada na literatura para este problema, no contexto de equalização multi-canal, é a utilização de predição multi-atraso (prediction multi-step) $[7,8]$. Uma outra possível solução pode ser baseada em equalizadores (filtros) mutuamente referenciados [9].

Neste artigo apresentamos uma nova solução usando um conjunto de FEPLLs, sendo que cada FEPLL apresenta um atraso distinto. A cada instante é selecionada a melhor saída do conjunto de FEPLLs, que pode ser usada como uma seqüência de treinamento para um equalizador largamente linear treinado. Este novo esquema de equalização apresenta alguma similaridade com as referências [7, 8, 9] citadas acima e com o trabalho apresentado em [10]. Os resultados de simulação mostram a viabilidade deste novo esquema de equalização. Seguindo a nomenclatura sugerida em [11] o equalizador proposto também poderia ser chamado de "super equalizador baseado em FEPLL".

Este artigo é organizado como segue. A Seção II apresenta os conceitos fundamentais do processamento largamente linear. A Seção III apresenta o FEPLL e o algoritmo LMS largamente linear de passo fixo empregado. $\mathrm{Na}$ Seção IV é detalhado o esquema de equalização proposto. A Seção V apresenta os resultados de simulação. A Seção VI concluí este artigo. 


\section{Processamento LARGAMENTE LinEAR: Conceitos FUNDAMENTAIS}

Considere uma seqüência $x[k]$ complexa, discreta, aleatória, com média nula. Usualmente, as estatísticas de segunda ordem de $x[k]$ são descritas pela função de autocovariância (FAC), que é definida por:

$$
\mu\left[k_{1}, k_{2}\right]=E\left\{x\left[k_{1}\right] x^{*}\left[k_{2}\right]\right\},
$$

onde ()$^{*}$ é a operação de conjugação complexa. Entretanto, tem sido observado que a FAC não é inteiramente suficiente para descrever as estatísticas de segunda ordem em alguns casos [6 - 9]. Logo, é necessário introduzirmos uma outra função chamada de pseudo-autocorrelação (FPAC), definida por:

$$
\rho\left[k_{1}, k_{2}\right]=E\left\{x\left[k_{1}\right] x\left[k_{2}\right]\right\} .
$$

Então, estas duas funções, $\mu\left[k_{1}, k_{2}\right]$ e $\rho\left[k_{1}, k_{2}\right]$, são necessárias para uma descrição completa das estatísticas de segunda ordem de $x[k]$. Quando $\rho\left[k_{1}, k_{2}\right]$ é igual a zero, a FPAC pode ser omitida e o processo é conhecido como próprio. Por outro lado, se o processo apresenta uma FPAC não nula, então este processo é dito ser impróprio. Um processo impróprio é estacionário no sentido amplo (widesense stationary - WSS) se, e somente se, $E\{x[k]\}=m_{x}$ é uma constante e tanto a FAC quanto a FPAC são independentes do tempo discreto $k[12,13]$.

Portanto, o processamento largamente linear faz o uso de uma observação complexa imprópria $x[k]$, e usa tanto $x[k]$ quanto o seu conjugado complexo $x^{*}[k]$.

\section{Modelo do Sistema e Filtro de ERro de PrediçÃo LARGAMENTE LINEAR}

\section{A. Modelo do Sistema}

Neste artigo, considera-se o sistema de comunicação digital como descrito pela Fig. 1, onde $a[k], d[k], n[k], x[k] \mathrm{e}$ $y[k]$ representam o sinal transmitido (pertencente a uma constelação real, com média nula), a saída do canal (complexo), o ruído (considerado complexo, próprio, com igual variância nas partes real e imaginária, aditivo, gaussiano e branco), o sinal recebido e a saída do equalizador, respectivamente.

O canal de comunicação digital, que pode ser representado por um filtro FIR (finite impulse response) de coeficientes complexos e comprimento $L$, apresenta os efeitos combinados do filtro de transmissão, do canal contínuo no tempo, filtro de recepção e da amostragem. Consideramos ainda que o transmissor gera uma seqüência de símbolos independentes identicamente distribuídos (i.i.d) e de variância $\sigma_{a}^{2}$. Já o ruído apresenta uma variância $\sigma_{n}^{2}$.

O sinal recebido, após uma amostragem à taxa de símbolo e perfeita sincronização de portadora, é dado por:

$$
x[k]=\sum_{m=0}^{L} h[m] a[k-m]+n[k],
$$

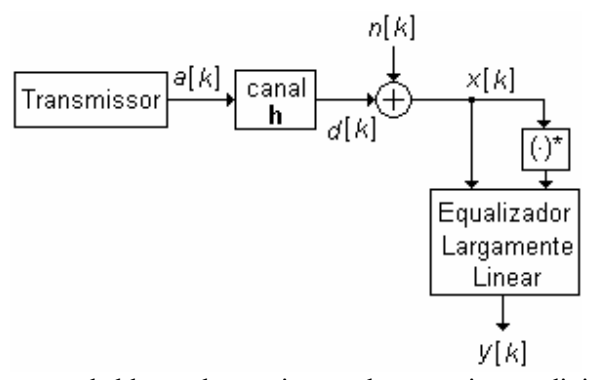

Fig. 1. Diagrama de blocos de um sistema de comunicação digital usando processamento largamente linear.

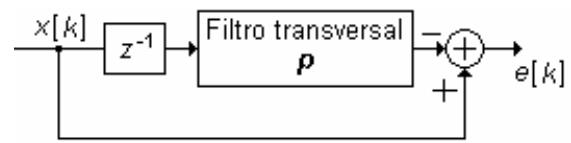

Fig. 2. Filtro de erro de predição linear.

onde $h[m]$ denota a resposta ao impulso do canal. Note que $x[k]$ é um processo impróprio e que o processamento largamente linear pode ser aplicado vantajosamente.

\section{B. Filtro de erro de predição linear (FEPL)}

O problema de predição linear consiste em prever o valor futuro de uma seqüência $X_{k}$ estacionária aleatória discreta no tempo, dado um conjunto de amostras passadas desta mesma seqüência [14]. Portanto, dados os valores $x[k], x[k-$ $1], \ldots, x[k-M+1]$ indicando as $M$ amostras passadas da seqüência até e incluindo o instante $k$, o problema de predição consiste em calcular o valor $\hat{x}\left[k+n \mid X_{k, k-M+1}\right]$ no instante de tempo $k+n, n \geq 1$. Podemos expressar o valor predito como uma função das amostras passadas por:

$$
\hat{x}\left[k+n \mid X_{k, k-M+1}\right]=F(x[k], \ldots, x[k-M+1]) .
$$

A predição é linear se a função $F\left({ }^{*}\right)$ pode ser expressa como uma combinação linear das amostras $x[k], x[k-1], \ldots$, $x[k-M+1]$. Quando a operação de predição linear envolve as amostras $x[k-1], x[k-2], \ldots, \quad x[k-M]$ para fazer uma estimativa de $x[k]$, dizemos que esta forma é uma predição linear direta de um passo (forward one-step linear prediction). Portanto, um preditor linear adaptativo de um passo pode ser descrito como um filtro FIR de ordem $M-1$ pela expressão

$$
\hat{x}[k]=\sum_{n=1}^{M} p_{n}^{*}[k] x[k-n],
$$

onde $p_{1}^{*}[k], \quad p_{2}^{*}[k], \ldots, \quad p_{M}^{*}[k]$ são os coeficientes do preditor no instante $k$. A diferença entre a amostra de entrada $x[k]$ e o valor predito $\hat{x}\left[k \mid X_{k-1, k-M}\right]$, é dada por

$$
e[k]=x[k]-\hat{x}\left[k \mid X_{k-1, k-M}\right] \text {, }
$$

e é conhecido como resíduo ou erro de predição direto (forward prediction error). O preditor ótimo, no sentido MSE (mean square error), é aquele que minimiza o valor médio quadrático de $e[k]$, isto é, os coeficientes $p_{n}{ }^{*}[k], n=$ $1, \ldots, M$, do preditor são ajustados tal que a função custo $J_{e}$ $=E\left\{|e[k]|^{2}\right\}$ seja minimizada. A Fig. 2 mostra o esquema de equalização usando um filtro de erro de predição linear. 


\section{Filtro de Erro de Predição Largamente Linear}

O FEPLL utiliza além de $x[k]$ seu conjugado complexo $x^{*}[k]$, como mostra a Fig. 3. De acordo com esta figura, o erro de predição largamente linear pode ser expresso por

$$
\begin{gathered}
e_{L L}[k]=x[k]-\sum_{n=1}^{M} f_{n}^{*}[k] x[k-n]-\sum_{n=1}^{M} g_{n}^{*}[k] x^{*}[k-n] \\
=x[k]-\boldsymbol{f}^{H}[k] \boldsymbol{x}[k-1]-\boldsymbol{g}^{H}[k] \boldsymbol{x}^{*}[k-1],
\end{gathered}
$$

onde $\quad \boldsymbol{x}[k-1]=[x[k-1] x[k-1] \ldots x[k-M]]^{T}$, $x[k]=\boldsymbol{h}^{T} \boldsymbol{a}[k]+n[k], \quad$ com $\quad \boldsymbol{h}=\left[\begin{array}{llll}h_{0} & h_{1} & \ldots & h_{L}\end{array}\right]^{T}$, $\boldsymbol{f}[k]=\left[f_{1}[k] \ldots f_{M}[k]\right]^{T}, \boldsymbol{g}[k]=\left[g_{1}[k] \ldots g_{M}[k]\right]^{T} \mathrm{e}$ $\boldsymbol{a}[k]=[a[k] \ldots a[k-L]]^{T}$. Omitindo a dependência temporal dos filtros $\boldsymbol{f}$ e $\boldsymbol{g}$, pode-se reescrever (7) como

$$
e_{L L}[k]=\mathbf{h}_{\mathbf{e}}^{T} \mathbf{a}_{\mathbf{e}}[k]-\mathbf{f}^{H} \mathbf{H} \mathbf{a}_{\mathbf{e}}[k]-\mathbf{g}^{H} \mathbf{H}^{*} \mathbf{a}_{\mathbf{e}}[k],
$$

onde $\mathbf{h}_{\mathbf{e}}=\left[\begin{array}{lllll}h_{0} \ldots & \ldots & h_{L} & 0 & \ldots\end{array}\right]^{T}$ tem dimensão $(M+L+1) \times 1$, $\mathbf{a}_{\mathbf{e}}=[a[k] \ldots a[k-L] \quad 0 \ldots 0]^{T}$ tem a mesma dimensão de $\mathbf{h}_{\mathbf{e}}$, e a matriz do canal de dimensão $M \times(M+L+1)$ é definida por:

$$
\mathbf{H}=\left[\begin{array}{cccccccc}
0 & h_{0} & h_{1} & \ldots & h_{L} & 0 & \ldots & 0 \\
0 & 0 & h_{0} & h_{1} & \ldots & h_{L} & \ddots & \vdots \\
\vdots & \vdots & \ddots & \ddots & \ddots & \ddots & \ddots & 0 \\
0 & 0 & \ldots & 0 & h_{0} & h_{1} & \ldots & h_{L}
\end{array}\right]
$$

note que a coluna de zeros da matriz $\mathbf{H}$ corresponde ao atraso de uma amostra. Aplicando o critério MMSE (minimum MSE) e as regras de derivação complexa [3, 14], podemos encontrar os filtros $\boldsymbol{f}$ e $\boldsymbol{g}$ ótimos pela resolução do seguinte sistema de equações:

$$
\left[\begin{array}{cc}
\mathbf{H H}^{H}+\xi \mathbf{I} & \mathbf{H H}^{\boldsymbol{T}} \\
\mathbf{H}^{*} \mathbf{H}^{H} & \mathbf{H}^{*} \mathbf{H}^{\boldsymbol{T}}+\xi \mathbf{I}
\end{array}\right]\left[\begin{array}{l}
\boldsymbol{f}_{\text {opt }} \\
\boldsymbol{g}_{\text {opt }}
\end{array}\right]=\left[\begin{array}{l}
\mathbf{H h}_{\mathbf{e}}^{H} \\
\mathbf{H}^{*} \mathbf{h}_{\mathbf{e}}^{H}
\end{array}\right],
$$

onde $\xi=\sigma_{n}^{2} / \sigma_{a}^{2}$ e I é uma matriz identidade de dimensão $M \times M$. Os vetores ótimos são dados por:

$$
\begin{aligned}
\boldsymbol{f}_{\text {opt }}= & {\left[\left(\mathbf{H H}^{\boldsymbol{H}}+\xi \mathbf{I}\right)-\mathbf{H} \mathbf{H}^{\mathbf{T}}\left(\mathbf{H}^{*} \mathbf{H}^{\boldsymbol{T}}+\xi \mathbf{I}\right)^{-1} \mathbf{H}^{*} \mathbf{H}^{\boldsymbol{H}}\right]^{-1} } \\
& \left(\mathbf{H h}_{\mathrm{e}}^{H}-\mathbf{H H}^{\boldsymbol{T}}\left(\mathbf{H}^{*} \mathbf{H}^{\boldsymbol{T}}+\xi \mathbf{I}\right)^{-1} \mathbf{H}^{*} \mathbf{h}_{\mathrm{e}}^{H}\right), \\
\boldsymbol{g}_{\text {opt }}= & {\left[\mathbf{H} \mathbf{H}^{\boldsymbol{T}}-\left(\mathbf{H} \mathbf{H}^{\boldsymbol{H}}+\xi \mathbf{I}\right)\left(\mathbf{H}^{*} \mathbf{H}^{H}\right)^{-1}\left(\mathbf{H}^{*} \mathbf{H}^{T}+\xi \mathbf{I}\right)\right]^{-1} } \\
& \left(\mathbf{H}^{*} \mathbf{h}_{\mathbf{e}}^{H}-\left(\mathbf{H} \mathbf{H}^{H}+\xi \mathbf{I}\right)\left(\mathbf{H}^{*} \mathbf{H}^{H}\right)^{-1} \mathbf{H} \mathbf{h}_{\mathbf{e}}^{H}\right) .
\end{aligned}
$$

Nota-se que $\boldsymbol{f}_{\text {opt }} \neq \boldsymbol{g}^{*}$ opt, o que é diferente do que ocorre no caso de equalização treinada quando $\boldsymbol{f}_{\text {opt }}=\boldsymbol{g}_{\text {opt }}$ [3]. Alternativamente, para o caso sem ruído $(\xi=0)$, pode-se reescrever o sistema de equações (10) como [5]:

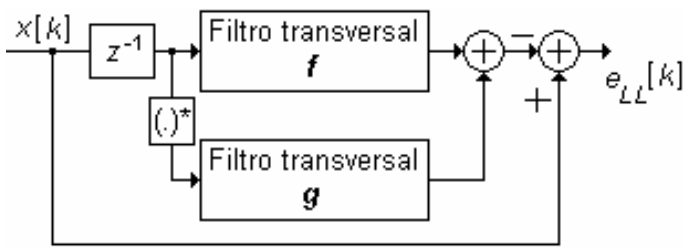

Fig. 3. Filtro de erro de predição largamente linear (FEPLL).

$$
\left[\begin{array}{ccccccc}
h_{0} & h_{0}^{*} & 0 & 0 & \cdots & 0 & 0 \\
\vdots & \vdots & h_{0} & h_{0}^{*} & \ddots & \vdots & \vdots \\
h_{L} & h_{L}^{*} & \vdots & \vdots & \ddots & 0 & 0 \\
0 & 0 & h_{L} & h_{L}^{*} & \ddots & h_{0} & h_{0}^{*} \\
\vdots & \vdots & \ddots & \ddots & \ddots & \vdots & \vdots \\
0 & 0 & \cdots & \cdots & \cdots & h_{L} & h_{L}^{*}
\end{array}\right]\left[\begin{array}{c}
f_{1} \\
g_{1} \\
\vdots \\
\vdots \\
f_{M} \\
g_{M}
\end{array}\right]=\left[\begin{array}{c}
h_{0} \\
\vdots \\
h_{L} \\
0 \\
\vdots \\
0
\end{array}\right] .
$$

A partir de (13) e usando os resultados gerais apresentados em [15] e [16], pode-se fazer as seguintes observações para que exista uma solução ótima ZeroForcing (ZF):

- o canal precisa ser complexo (ao menos um coeficiente precisa ser complexo);

- os canais h e $\mathbf{h}^{*}$ não podem possuir zeros em comum (para satisfazer a identidade de Bezout);

- os filtros ótimos $\boldsymbol{f}_{\text {opt }}$ e $\boldsymbol{g}_{\text {opt }}$ precisam ter somente L-1 coeficientes;

- o FEPLL é um equalizador do tipo ZF, a menos de uma constante multiplicativa, isto é, $e_{L L}[k]=h_{0} a[k]$.

\section{Algoritmo Adaptativo de passo fixo}

A seguir, será apresentado um algoritmo adaptativo para o cálculo dos coeficientes do FEPLL. A partir de (7) pode-se derivar um algoritmo LMS largamente linear usando as regras de derivação complexa convencionais [14]. Assim, pode-se calcular:

$$
\begin{aligned}
\boldsymbol{f}[k+1] & =\boldsymbol{f}[k]-\mu \frac{\partial\left|e_{L L}[k]\right|^{2}}{\partial \boldsymbol{f}^{*}[k]} \\
& =\boldsymbol{f}[k]-\mu e_{L L}^{*}[k] \boldsymbol{x}[k], \\
\boldsymbol{g}[k+1] & =\boldsymbol{g}[k]-\mu \frac{\partial\left|e_{L L}[k]\right|^{2}}{\partial \boldsymbol{g}^{*}[k]} \\
& =\boldsymbol{g}[k]-\mu e_{L L}^{*}[k] \boldsymbol{x}^{*}[k],
\end{aligned}
$$

onde $\mu$ é passo do algoritmo LMS. Para compensar o efeito multiplicativo (ajustes de fase e ganho) provocado pelo coeficiente $h_{0}$ do canal, pode-se usar o seguinte procedimento também adaptativo:

$$
\begin{gathered}
\Phi_{k+1}=\Phi_{k}+\mu_{\Phi} \operatorname{angle}\left(e_{L L}[k] \exp \left(j \Phi_{k}\right)\right), \\
G_{k+1}=G_{k}+\mu_{G}\left(E\left\{|a[k]|^{2}\right\}-|y[k]|^{2}\right), \\
\gamma_{k}=\sqrt{\left|G_{k}\right|},
\end{gathered}
$$




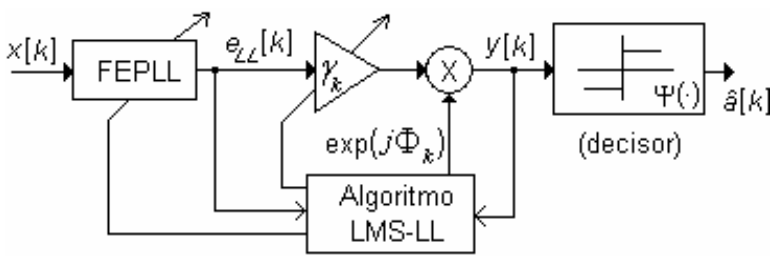

Fig. 4. Diagrama de blocos de um FEPLL adaptativo de atraso zero com compensação de ganho e fase e um decisor não-linear.

onde $\mu_{\Phi}$ e $\mu_{G}$ são os passos de adaptação da fase e do ganho, respectivamente. $\mathrm{O}$ valor inicial do ajuste de fase é zero $\left(\Phi_{0}=0\right)$ e do ajuste do ganho pode ser unitário $\left(G_{0}=1\right)$. Inicialmente, os coeficientes dos filtros $\boldsymbol{f}$ e $\boldsymbol{g}$ podem ser todos nulos, isto é, $\boldsymbol{f}[0]=\boldsymbol{g}[0]=\mathbf{0}$. Esta inicialização não é crítica.

As expressões (7) e de (14) a (18) completam o algoritmo LMS largamente linear para o FEPLL usando passos fixos. A Fig. 4 mostra o diagrama de blocos do FEPLL adaptativo. Este equalizador pode ser divido em três partes: o FEPLL, controle de ganho e fase e o decisor. O decisor $\Psi(\bullet)$ é um dispositivo não-linear, sem memória, que mapeia a saída $y[k]$ do equalizador no símbolo da constelação utilizada (4PAM, por exemplo) mais próximo. Note que a saída $y[k]$ pode ser descrita simplesmente por

$$
y[k]=a[k]+n_{f e}[k],
$$

onde $n_{f e}[k]$ representa o ruído filtrado e o erro na estimativa de $h[0]$ combinados.

\section{ESQUEMA PRoposto}

Como descrito em [7] e [8], a predição multi-passo (ou multi-atraso) pode ser usado para equalização cega com um atraso de decisão arbitrário em sistemas SIMO. Na ausência de ruído, o erro de predição multi-passo pode ser modelado como a saída de um canal truncado. Assim, para um FEPLL com um passo $\Delta>1$ (FEPLL “ $\Delta$-step") pode-se obter:

$$
e_{\Delta}[k]=\sum_{m=0}^{\Delta-1} h[m] a[k-m]
$$

Se for usado, em cascata, um filtro de erro de predição reverso (backward linear prediction) largamente linear de ordem suficiente, o erro de predição ótimo torna-se $[7,8]$ :

$$
e_{B}[k]=h[\Delta-1] a[k-\Delta+1] \text {. }
$$

A grande vantagem deste método é que se pode tentar selecionar o coeficiente de maior energia do canal e, assim, facilitar a correta estimação do símbolo transmitido (com um atraso igual a $\Delta-1$ ). A solução expressa por (20) pode ser obtida de uma forma alternativa, como será descrito a seguir, usando-se apenas FEPLLs.

Considerando-se uma alta relação sinal ruído, no FEPLL descrito na seção anterior (com $\Delta=1$, atraso zero), é possível se conseguir uma boa estimativa de $h[0]$ e $\hat{a}[k]$, sendo $o$ erro de decisão direta é expresso por $\varepsilon_{D D 0}=\hat{a}[k]-\mathfrak{R}(y[k])$, onde $\mathfrak{R}$ indica a parte real.
Quando ocorre a convergência do algoritmo, após um número suficiente de amostras (o que vai depender do canal a ser equalizado) [5], o erro de decisão direta se torna pequeno, $\hat{a}[k]=a[k]$, o valor estimado de $h[0]$ no instante $k$ é dado por

$$
\hat{h}[0]=\exp \left(-j \Phi_{k}\right) / \sqrt{\gamma_{k}} \approx h[0]
$$

e a saída do FEPLL, sem a correção de ganho ou fase, é

$$
e_{L L 0}[k]=h[0] a[k]+n_{1}[k] \text {, }
$$

onde $n_{1}[k]$ é o ruído na saída do filtro. O símbolo decidido pode ser calculado por:

$$
\hat{a}[k]=\Psi\left(y_{0}[k]\right)=\Psi\left(e_{L L 0}[k] / \hat{h}[0]\right),
$$

onde $y_{0}[k]$ é a saída deste primeiro FEPLL. Na saída do FEPLL com $\Delta=2$, obviamente sem qualquer ajuste de fase ou ganho, tem-se

$$
e_{L L 1}[k]=h[0] a[k]+h[1] a[k-1]+n_{2}[k],
$$

logo, calculando

$$
e_{1 R}[k]=e_{L L 1}[k]-e_{L L 0}[k],
$$

resulta em $h[1] a[k-1]$ mais ruído. Se o erro de decisão direta $\left(\varepsilon_{D D}\right)$ do primeiro FEPLL estiver abaixo de um certo limiar, é mais conveniente calcular

$$
\begin{array}{r}
e_{1 R}[k]=e_{L L 1}[k]-\hat{h}[0] \hat{a}[k] \\
=h[1] a[k-1]+n_{1 R}[k],
\end{array}
$$

pois não será adicionado o ruído do estágio anterior. $\mathrm{Na}$ saída deste FEPLL, o símbolo será decidido com base em $e_{1 R}[k]$ e $\hat{h}[1]$ :

$$
\hat{a}[k-1]=\Psi\left(y_{1}[k]\right)=\Psi\left(e_{1 R}[k] / \hat{h}[1]\right),
$$

onde $y_{1}[k]$ é saída deste segundo FEPLL. O erro de decisão para este estágio, no instante $k$, é expresso por:

$$
\varepsilon_{D D 1}=\hat{a}[k-1]-\mathfrak{R}\left(y_{1}[k]\right)
$$

onde $\hat{h}[1]$ é uma estimativa de $h[1]$. Todo esse procedimento está esquematizado na Fig. 5.

Na saída de um FEPLL com $\Delta=3$ tem-se:

$$
e_{L L 2}[k]=\sum_{m=0}^{2} h[0] a[k-m]+n_{2}[k]
$$

usando-se os resultados e procedimentos anteriores, é possível se estimar $h[2]$ e $a[k-2]$ : 


$$
\begin{gathered}
e_{2 R}[k]=e_{L L 2}[k]-\hat{h}[0] a[k]-\hat{h}[1] a[k-1] \\
=h[2] a[k-2]+n_{2 R}[k] .
\end{gathered}
$$

Logo, com estes três filtros pode-se estimar $a[k], a[k-1]$, $a[k-2]$ e os coeficientes do canal $h[0], h[1]$ e $h[2]$. De uma forma geral, tendo-se vários FEPLLs com atrasos $0,1, \ldots, D$, pode-se estimar os coeficientes $h[0], \ldots, h[D]$, e os símbolos $a[k], \ldots, a[\mathrm{k}-D]$. Os símbolos estimados, depois de alinhados apropriadamente, podem ser combinados para gerar uma estimativa mais robusta de $a[k-D]$. Por exemplo, no instante $k$, pode-se calcular:

$$
y_{R}[k]=\frac{\frac{e_{L L 0}[k-D]}{\hat{h}[0]}+\frac{e_{1 R}[k-D+1]}{\hat{h}[1]}+\cdots+\frac{e_{D R}[k]}{\hat{h}[D]}}{D+1}
$$

que leva em conta a contribuição de cada FEPLL. Após o alinhamento de todas as saídas, pode-se, ainda, usar o seguinte critério para tornar o procedimento mais robusto: em cada instante $k$, a saída selecionada será aquela que apresentar menor erro de decisão direta dentre as saídas disponíveis.

Finalmente, esta saída selecionada (saída ótima) é usada como uma seqüencia de treinamento para um equalizador LMS largamente linear treinado de passo fixo $\mu_{t}[3,17]$. Quando o erro de decisão direta deste equalizador treinado ficar abaixo do valor limiar $(<0,85)$, ele passa a operar no modo de decisão direta. Desta forma, o desempenho final do sistema, para uma relação sinal-ruído apenas moderada ( $15 \mathrm{~dB})$, pode se aproximar muito daquele que seria obtido por uma equalização largamente linear treinada convencional, como será mostrado na próxima seção através de simulações. A Fig. 6 mostra o esquema final de equalização proposto, com a omissão de alguns detalhes já apresentados na Fig. 5. Note que o sinal equalizado é $y_{t}[k]$ e o símbolo decidido é $\hat{a}[k]=\Psi\left(y_{t}[k]\right)$.

\section{Resultados de Simulação}

Nas simulações de Monte Carlo consideradas a seguir, o sinal transmitido pertence a uma constelação 4-PAM. Os canais e os parâmetros $\left(\mu_{L L}, \mu_{\Phi}, \mu_{G}\right.$ e $\left.\mu_{t}\right)$ dos algoritmos usados nas simulações estão indicados na Tabela I. Estes canais são complexos, de fase não-mínima e apresentam um ou mais zeros próximos ao círculo de raio unitário.

O banco de FEPLLs simulado usa apenas 3 FEPLLs (atrasos de 0,1 e 2 amostras) e um equalizador LL treinado com a saída ótima dos FEPLLs, sendo esta, após o alinhamento dos símbolos, uma versão atrasada de 4 amostras em relação ao sinal original. Tanto os FEPLLs quanto o equalizador LL têm 12 coeficientes $(M=6)$. Para efeitos comparativos, foi realizada a equalização treinada dos mesmos canais por um equalizador LL, também com um atraso de equalização de 4 amostras.

É sabido que este atraso pode influenciar no desempenho do sistema [3, 11, 17], mas para uma equalização com um equalizador largamente linear, da mesma forma que uma equalização usando super-amostragem (sistemas polifásicos), um atraso de equalização igual a $(L+M) / 2$ é razoável [11].

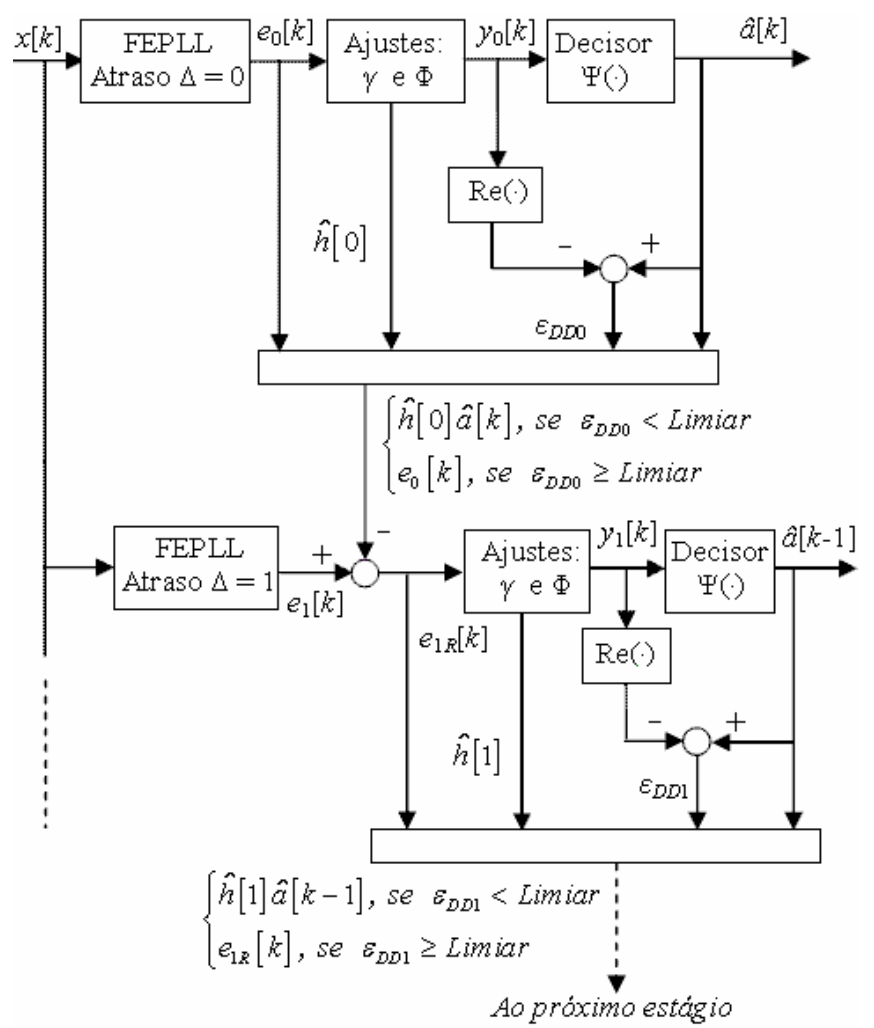

Fig. 5. Diagrama de blocos dos dois primeiros estágios do esquema de equalização proposto usando FEPLL adaptativo.

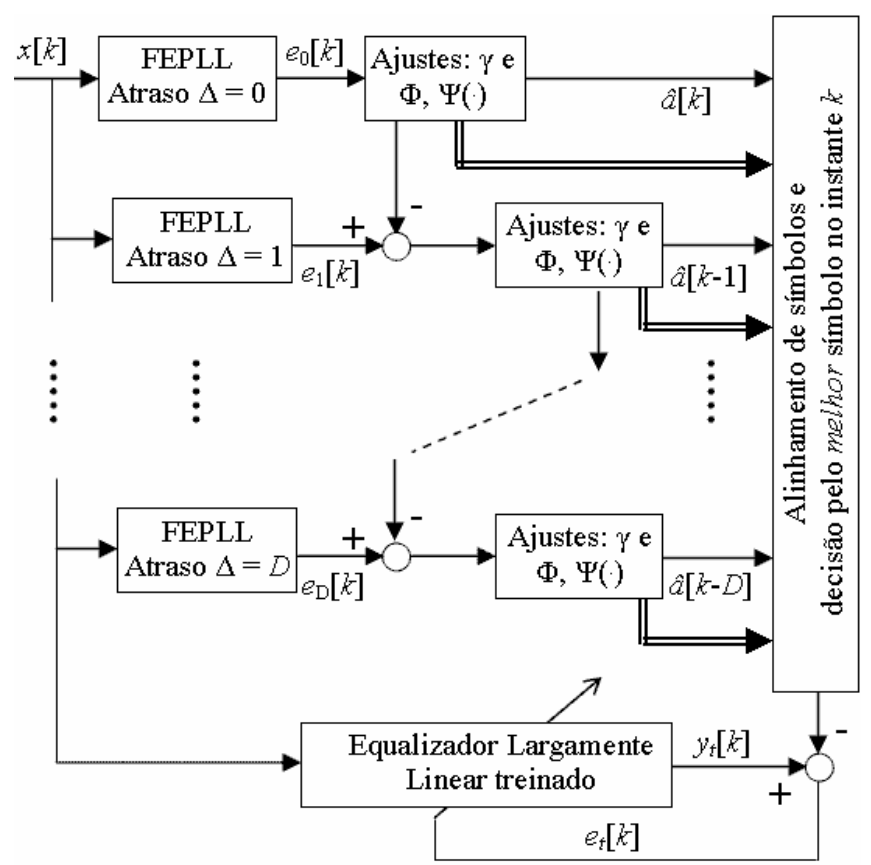

Fig. 6. Diagrama de blocos completo do esquema de equalização proposto.

A Fig. 7 mostra o efeito do atraso de equalização para o Canal 1 (Tabela I) na variância do erro, definida como

$$
V_{\text {erro }}=10 \log _{10}\left(E\left\{\left|a[k]-y_{t}[k]\right|^{2}\right\}\right) \text {, }
$$

na saída de um equalizador LL treinado de 12 coeficientes considerando-se relações sinal-ruído de 10, 20 e 30 dB. Para os demais canais simulados o resultado é bastante similar. A partir da Fig. 7, é fácil verificar que o atraso de equalização poderia ficar entre 3 e 7 amostras, sendo 4 amostras o valor ótimo para o Canal 1. 
A avaliação da taxa de erro de símbolo (TES) é feita após a um período de treinamento realizado com as primeiras 7500 amostras ótimas geradas pelo banco de FEPLLs. O equalizador largamente linear trabalha com um algoritmo LMS de passo fixo, já que esta configuração é a mais simples e apresenta um desajuste final menor que sua versão de passo normalizado [17]. A TES para os três canais é mostrada na Fig. 8. A equalização dos canais 1 e 2 apresenta um comportamento semelhante, já o canal 3 é mais difícil de ser equalizado e somente quando a relação sinal-ruído é igual ou maior que $15 \mathrm{~dB}$ é que a TES se torna essencialmente igual ao caso treinado.

TABELA I

COEFICIENTES DOS CANAIS

\begin{tabular}{|c|c|c|c|}
\hline$h$ & Canal 1 & & 3 \\
\hline$h[0]$ & $0,4133+0,4724 j$ & $0,6950-0,0695 i$ & $0,2783-0,2087$ \\
\hline$h[1]$ & $0,2952+0,4133 j$ & $0,1286+0,2328 j$ & $0,3478-0,2783 j$ \\
\hline$h[2]$ & $0,3543-0,3897 j$ & $0,3708-0,4688 j$ & $0,5218+0,3478 j$ \\
\hline$h[3]$ & $0,1181+0,1771 j$ & $0,1078-0,2168 j$ & $-0,3478-0,2609 j$ \\
\hline$h[4]$ & $-0,1181+0,1063 j$ & $0,1136-0,1119 j$ & $0,1739+0,2609 j$ \\
\hline \multirow{4}{*}{ 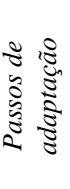 } & $\mu_{L L}=0,0001$ & $\mu_{L L}=0,0001$ & $\mu_{L L}=0,0002$ \\
\hline & $\mu_{\Phi}=0,001$ & $\mu_{\Phi}=0,001$ & $\mu_{\Phi}=0,001$ \\
\hline & $\mu_{G}=0,01$ & $\mu_{G}=0,01$ & $\mu_{G}=0,01$ \\
\hline & $\mu_{t}=0,001$ & $\mu_{t}=0,001$ & $\mu_{t}=0,001$ \\
\hline
\end{tabular}

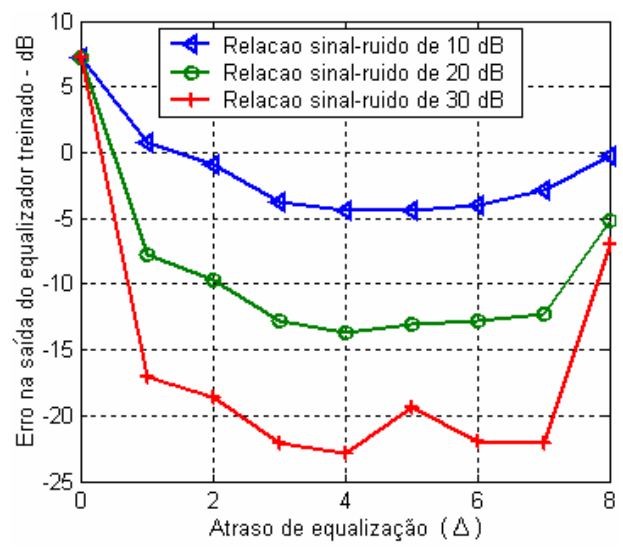

Fig 7. Variância do erro (em dB) na saída do equalizador LL treinado em função do atraso de equalização para o Canal 1.
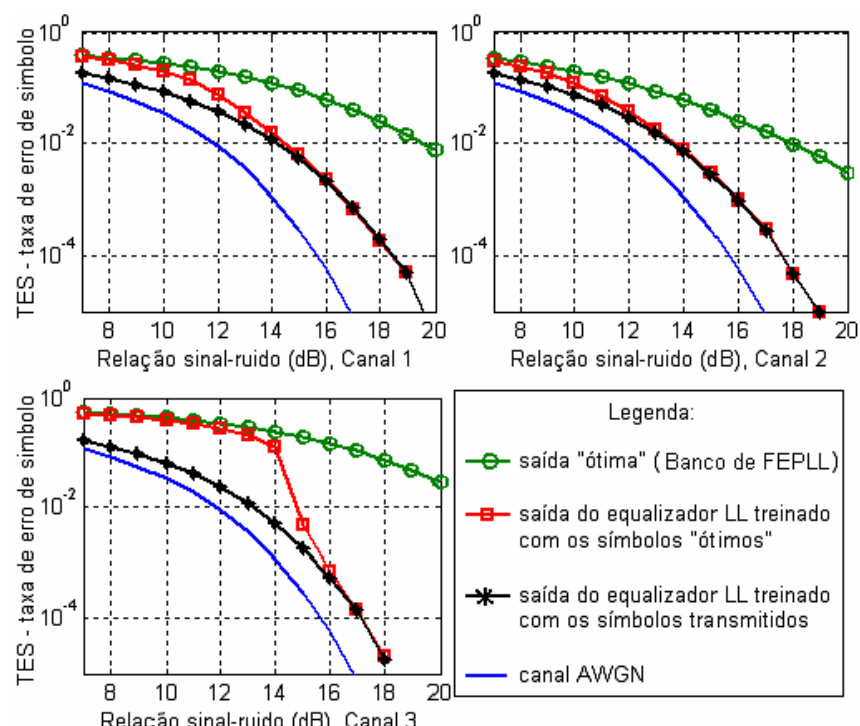

Fig 8. Taxa de erro de símbolo (TES) para os canais simulados.

\section{CONCLUSÃo}

Neste artigo foi apresentado um novo esquema de equalização cega usando um conjunto de FEPLLs que gera uma seqüência de treinamento para um equalizador largamente linear. Naturalmente, este esquema de equalização apresenta uma complexidade computacional algumas vezes (nas simulações apresentadas, cerca de quatro vezes) superior àquela que seria obtida por um único FEPLL. Entretanto, o esquema realiza uma equalização mais robusta do canal e pode apresentar uma TES similar a de uma equalização treinada quando a relação sinal-ruído é maior ou igual a $15 \mathrm{~dB}$.

\section{REFERÊNCIAS}

[1] J. G. Proakis, "Digital Communications", McGraw-Hill, 3a. Edição, 1995.

[2] G. Gelli, L. Paura and A. R. P. Ragozini, "Blind widely linear multiuser detection," IEEE Commun. Lett., vol. 4, no. 6, pp. 187-189, Jun. 2000.

[3] W. H. Gerstacker, R. Schober and A. Lampe, "Receivers with widely linear processing for frequency-selective channels," IEEE Commun. Lett., vol. 7, no. 9, pp. 1512-1523, Sep. 2003.

[4] R. Schober, W. H. Gerstacker, L. H.-J. Lampe, "Data-Aided and Blind Stochastic Gradient Algorithms for Widely Linear MMSE MAI Suppression for DS-CDMA", IEEE Trans. on Signal Processing, vol. 52, pp. 746-755, no. 3, Mar 2004.

[5] F. J. A. de Aquino, C. A. F. da Rocha, L. S. Resende, "Widely Linear Prediction Applied to Blind Equalization", Proceedings of International Workshop on Telecommunications (IWT) 2007, pp. 240-247, Feb. 2007

[6] B. Picinbono, e P. Chevalier, "Widely linear estimation with complex data," IEEE Trans. Signal Processing, vol. 43, pp. 2030-2033, Aug. 1995.

[7] D. Gesbert e P. Duhamel. "Robust blind channel identification and equalization based on multi-step predictors". In Proc. of Int. Conf. on Acoust. Speech and Signal Proc., pages 3621-3624, 1997.

[8] D. T. Slock e C. B. Papadias. "Further results on blind identification and equalization of multiple fir channels". In Proc. of Int. Conf. on Acoust. Speech and Signal Proc., pages 1964-1 967, Detroit, MI, May, 1995.

[9] D. Gesbert. P Duhamel. e S. Mayrargue, "On-line blind multichannel equalization based on mutually referenced filters," IEEE Trans. Signal Proc., vol. 45, pp. 2307-2317, Sept. 1997.

[10] K. S. Ahn, J. Cho, e H. Ki Baik, "FPEF-Based Blind Adaptive Channel Equalization with Arbitrary Decision Delay", IEEE Trans. on Consumer Electronics, Vol. 50, No. 4, pp. 984-990, Nov. 2004.

[11] D. Gesbert. A.-J. van der Veen, e A. Paulraj, "On the equivalence of blind equalizers based on MRE and subspace intersections," IEEE Trans. Signal Proc., vol. 45, no. 3, pp. 856-859, Mar. 1999.

[12] Neeser, F. D. e Massey, J. L. "Proper complex random processes with applications to information theory," IEEE Trans. Inform. Theory, vol. 39, pp. 1293-1302, July 1993.

[13] B. Picinbono, P. Bondon, "Second-order statistics of complex signals", IEEE Transactions on Signal Processing, Vol. 45, pp. 411420, Feb. 1997.

[14] S. Haykin, "Adaptive Filter Theory", $4^{\text {th }}$. ed., Prentice Hall, New Jersey, 2002.

[15] C.B. Papadias e D.T.M. Slock, "Fractionally spaced equalization of linear polyphase channels and related blind techniques based on multichannel linear prediction", IEEE Trans. on Signal Processing, Vol. 47, pp. 641-654, Mar. 1999.

[16] G.B. Giannakis, and S.D. Halford, "Blind fractionally spaced equalization of noisy FIR channels: direct and adaptive solutions", IEEE Trans. on Signal Processing, vol. 45, pp.:2277 - 2292, Sept. 1997.

[17] F.J.A. de Aquino, C. A. F. da Rocha e L. S. Resende, "Accelerating the Convergence of the Widely Linear LMS Algorithm for Channel Equalization", VI International Telecommunications Symposium (ITS2006), Fortaleza-CE, Brazil, vol. 1, 57-61, Sep., 2006. 